\title{
ON A PROBLEM IN THE THEORY OF PARTITIONS ${ }^{1}$
}

\author{
E. G. STRAUS
}

In this note we give an affirmative answer to a problem posed by Sherman K. Stein (Bull. Amer. Math. Soc. 66 (1960), 510). That is, we show that for every number $m, 1 \leqq m \leqq p(n)$ where $p(n)$ is the number of partitions of the positive integer $n$, there exists a set $A(m, n)$ of positive integers such that $n$ has $m$ partitions into elements of $A(m, n)$. Our result generalizes to the case of partitions of vectors with nonnegative integral components.

Definition 1. Let $S=\left\{s_{1}, s_{2}, \cdots, s_{k}\right\}$ be a set of positive integers; then $p(S, n)$ denotes the number of partitions of $n$ into elements of $S$. In particular we define $p(S, 0)=1$ and $p(S, n)=0$ for $n<0$.

Lemma 1.

$$
p(S, n)=\sum_{i=1}^{k} p\left(S-S_{i}, n-s_{i}\right)
$$

where $S_{i}=\left\{s_{1}, s_{2}, \cdots, s_{i-1}\right\}, S_{1}=\varnothing$. (Note: this lemma is independent of the ordering of $S$.)

Proof. The $i$ th term in the sum counts the number of partitions of $n$ into elements of $S$ where the first term occurring is $s_{i}$.

COROLLARY 1. If $1 \in S$ then $p(S, n)$ is a nondecreasing function of $n$.

Corollary 2.

$$
p(S, n) \leqq \sum_{l=0}^{n-1} p(S, l) .
$$

Proof. By Lemma 1

$$
\begin{aligned}
p(S, n) & =\sum_{i=1}^{k} p\left(S-S_{i}, n-s_{i}\right) \\
& \leqq \sum_{i=1}^{k} p\left(S, n-s_{i}\right) \\
& \leqq \sum_{l=0}^{n-1} p(S, l) .
\end{aligned}
$$

Received by the editors January 9, 1961.

1 This work has been supported in part by a grant from the National Science Foundation. 
Corollary 3. If $1 \in S$ then $p(S, n+1) \leqq p(S, n)+p(S, n-1)$.

Proof. We use simultaneous induction on $n$ and $k$, the number of elements of $S$. If $n=0$ then

$$
p(S, 1) \leqq 1=p(S, 0)=p(S, 0)+p(S,-1)
$$

for all $S$. If $k=1$ then $S=\{1\}$ and

$$
p(\{1\}, n+1)=1=p(\{1\}, n) \leqq p(\{1\}, n)+p(\{1\}, n-1)
$$

for all nonnegative $n$.

Now assume we had the minimal $n$ and a minimal $S$ for which the result is false then there is an $s \in S, s \neq 1$ and by Lemma 1

$$
\begin{aligned}
p(S, n+1)= & p(S, n+1-s)+p(S-\{s\}, n+1) \\
\leqq & p(S, n-s)+p(S, n-1-s)+p(S-\{s\}, n) \\
& +p(S-\{s\}, n-1) \\
\leqq & p(S, n)+p(S, n-1) .
\end{aligned}
$$

LEMMA 2. If $1=a_{1} \leqq a_{2} \leqq \cdots . a_{k} \leqq \cdots$ and $a_{k+1} \leqq 2 a_{k}$ where the $a_{k}$ are integers, then for every integer $n$ with $1 \leqq n \leqq a_{1}+a_{2}+\cdots+a_{k}$ there exist indices $1 \leqq i_{1}<i_{2}<\cdots<i_{j} \leqq k$ so that $n=a_{i_{1}}+\cdots+a_{i j}$.

Proof. By induction on $k$. If $k=1$ then $n=1=a_{1}$. Assume the lemma proved for $k-1$. If $n \leqq a_{1}+\cdots+a_{k-1}$ then the lemma is true by the induction hypothesis. If $n>a_{1}+\cdots+a_{k-1}$ then

$$
n>a_{k}\left(\frac{1}{2}+\frac{1}{4}+\cdots+\frac{1}{2^{k-1}}\right)=a_{k}\left(1-\frac{1}{2^{k-1}}\right) \geqq a_{k}-1
$$

so that $n \geqq a_{k}$. Thus $0 \leqq n-a_{k} \leqq a_{1}+\cdots+a_{k-1}$ and the lemma holds, by the induction hypothesis.

LEmma 3. If $1 \in S$ then the sequence $p(S, 1), p(S, 2), \cdots, p(S, k), \cdots$ satisfies the hypothesis of Lemma 2.

Proof. We have $p(S, 1)=1$ and $p(S, k+1) \geqq p(S, k)$ by Corollary 1 ; finally $p(S, k+1) \leqq 2 p(S, k)$ by Corollary 3 of Lemma 1 .

LEMMA 4. If $S \subset\{1,2, \cdots,[n / 2]\}$ and $T \subset\{[n / 2]+1, \cdots, n\}$ then $p(S \cup T, n)=p(S, n)+\sum_{t \in T} p(S, n-t)$.

Proof. Since $t+t^{\prime}>n$ for any $t, t^{\prime} \in T$ a partition of $n$ into elements of $S \cup T$ is either a partition into elements of $S$ alone or $t$ plus a partition of $n-t$ into elements of $S$.

CoROLlaRY. If $1 \in S$ and $S \subset\{1,2, \cdots,[n / 2]\}$ then for any integer $m$ with 


$$
\begin{aligned}
p(S, n) & \leqq m \\
& \leqq p(S, n)+\sum_{l=0}^{[(n-1) / 2]} p(S, l)
\end{aligned}
$$

there exists a set $T \subset\{[n / 2]+1, \cdots, n\}$ so that $p(S \cup T, n)=m$.

LEMMA 5.

$$
p\left(S \cup\left\{\left[\frac{n}{2}\right]\right\}, n\right) \leqq 1+p(S, n)+\sum_{l=0}^{[(n-1) / 2]} p(S, l)
$$

Proof. If $[n / 2] \in S$ the result is obvious. If $[n / 2] \notin S$ then

$$
\begin{aligned}
p\left(S \cup\left\{\left[\frac{n}{2}\right]\right\}\right. & , n) \\
& =p(S, n)+p\left(S, n-\left[\frac{n}{2}\right]\right)+p\left(S, n-2\left[\frac{n}{2}\right]\right) \\
& \leqq 1+p(S, n)+p\left(S,\left[\frac{n-1}{2}\right]+1\right)
\end{aligned}
$$

so that the lemma reduces to

$$
p(S, m+1) \leqq \sum_{l=0}^{m} p(S, l), \quad m=\left[\frac{n-1}{2}\right] ;
$$

which is Corollary 2 of Lemma 1.

LEMma 6. If $1 \in S$ and $S \subset\{1,2, \cdots, m-1\}$ where $m<[n / 2]$ then

$$
\begin{aligned}
p(S \cup\{m\}, n) \leqq & 1+p\left(S \cup\left\{m+1, \cdots,\left[\frac{n}{2}\right]\right\}, n\right) \\
& +\sum_{l=0}^{[(n-1) / 2]} p\left(S \cup\left\{m+1, \ldots\left[\frac{n}{2}\right]\right\}, l\right) \\
= & 1+p(S \cup\{m+1, \cdots, n\}, n) .
\end{aligned}
$$

Proof. The hypothesis implies $m \geqq 2$. By repeated application of Lemma 1 we get

$$
p(S \cup\{m\}, n)=\sum_{l=0}^{[n / m]} p(S, n-l m)
$$

and by Corollary 3 of Lemma 1 together with Lemma 1 


$$
\begin{aligned}
& \sum_{l=0}^{[n / m]} p(S, n-l m) \\
& \quad \leqq p\left(S, n-\left[\frac{n}{m}\right] m\right)+p(S, n)+\sum_{l=1}^{[n / m]-1} p(S, n-l m) \\
& \quad \leqq p\left(S, n-\left[\frac{n}{m}\right] m\right)+p(S, n) \\
& \quad+\sum_{l=1}^{[n / m]-1}(p(S, n-l m-1)+p(S, n-l m-2)) \\
& \leqq \\
& \quad+p(S, n)+\sum_{l=0}^{n-m-1} p(S, l) \\
& \leqq \\
& \quad 1+p(S, n)+\sum_{k=1}^{n-m} p(S \cup\{m+1, \cdots, m+k\}, n-m-k) \\
& =1+p(S \cup\{m+1, \cdots, n\}, n) .
\end{aligned}
$$

ThEOREM 1. For every integer $m, 1 \leqq m \leqq p(n)$, there exists a set $S \subset\{1,2, \cdots, n\}$ such that $p(S, n)=m$.

Proof. We construct a sequence $S_{1}, S_{2}, \cdots, S_{n}$ of subsets of $\{1,2, \cdots,[n / 2]\}$ as follows:

(1) $S_{1}=\{1\}, \quad S_{2}=\{1,[n / 2]\}$.

(2) Let $r$ be the maximal element $\leqq[n / 2]$ which is not contained in $S_{i}$ then $S_{i+1}=S_{i} \cup\{r\}-\{r+1, \cdots,[n / 2]\}$.

It is clear that this sequence terminates only with $S_{N}$ $=\{1,2, \cdots,[n / 2]\}$. According to the corollary to Lemma 4 the numbers $p\left(S_{i} \cup T, n\right)$, where $T \subset\{[n / 2]+1, \cdots, n\}$, fill the interval $I_{i}$ of numbers $p\left(S_{i}, n\right) \leqq m \leqq p\left(S_{i} \cup\{[n / 2]+1, \cdots, n\}, n\right)$. By Lemmas 5 and 6 the union $J_{i}=I_{1} \cup I_{2} \cup \ldots \cup I_{i}$ is itself an interval so that $J_{N}$ contains all $m$ with $1 \leqq m \leqq p(n)$.

One might ask whether our theorem has an analogue if $p(n)$ is replaced by $p(S, n)$, that is whether for any $m$ with $1 \leqq m \leqq p(S, n)$ there is a subset $S^{\prime}$ of $S$ so that $p\left(S^{\prime}, n\right)=m$. The answer is obviously no, since $p(\{1,2\}, n)=[n / 2]+1$ while $p(\{1\}, n)=1$ and $p(\{2\}, n)$ $\leqq 1 .^{2}$ It is, however, possible to generalize our result to the partition of vectors.

Definition. Let $n=\left(n_{1}, n_{2}, \cdots, n_{k}\right)$ where the $n_{i}$ are integers; then $p_{k}(n)$ is the number of partitions of $n$ into $k$-vectors whose components are nonnegative integers not all of which are zero.

THEOREM 2. Let $m$ be an integer, $1 \leqq m \leqq p_{k}(n)$ then there exists a set

2 The answer is yes if we let $S=\{m, m+1, m+2, \cdots\}$ be the set of all integers $\geqq m, m$ a positive integer, without appreciable change in the proof. 
$S$ of nonnegative nonzero k-vectors such that the number $p_{k}(S, n)$ of partitions of $n$ into elements of $S$ is $m$.

The proof involves only simple modifications of the steps which led to Theorem 1 . We outline them here. Lemma 1 remains valid. In Corollary 1 the condition $1 \in S$ is to be replaced by the condition that $S$ contains all the unit-vectors. By "nondecreasing" we now refer to the partial ordering given by $a<b$ if $b-a$ has nonnegative components. In Corollary 2 the summation is understood to be over all vectors which precede $n$ in the above sense.

In Corollary 3 the vectors $n+1$ and $n-1$ must be understood as the result of adding and subtracting from $n$ the same (arbitrary) unit vector. We need a slight modification of this result:

If $S$ contains all unit vectors and $n+1=n^{\prime}+1^{\prime}$ where 1 and $1^{\prime}$ are two different unit vectors then

$$
p_{k}(S, n+1) \leqq p_{k}(S, n)+p_{k}\left(S, n^{\prime}\right) .
$$

Instead of the subsequent lemmas we get

LEMмA $3^{\prime}$. If $S$ contains the unit vectors then the set $\left\{p_{k}(S, l)\right\}$ where $l$ ranges over all nonnegative vectors with $l<n$ can be arranged as a nondecreasing sequence which satisfies the hypothesis of Lemma 2.

LEMma $4^{\prime}$. If $S \cap T=\varnothing$ and $2 t>n$ for every $t \in T$, then

$$
p_{k}(S \cup T, n)=p_{k}(S, n)+\sum_{t \in T} p_{k}(S, n-t) .
$$

Corollary. Let $S$ contain the unit vectors and $S \cap T=\varnothing$ where $T$ is the set of all vectors $t$ which satisfy $2 t>n$. Then for every integer $m$ with $p_{k}(S, n) \leqq m \leqq p_{k}(S \cup T, n)$ there exists a $T^{\prime} \subset T$ so that $p_{k}\left(S \cup T^{\prime}, n\right)$ $=m$.

Lemma $5^{\prime}$. Let $n^{(i)}=\left(n_{1}, n_{2}, \cdots,\left[n_{i} / 2\right], \cdots, n_{k}\right)$ and let $T$ be as in the preceding corollary. Then

$$
p_{k}\left(S \cup\left\{n^{(i)}\right\}, n\right) \leqq 1+p_{k}(S \cup T, n) .
$$

LEMMA 6'. Let $T$ be as above and let $S$ contain the unit vectors with $S \cap T=\varnothing$. Let $m \notin S \cup T$ be a vector all whose successors are contained in $S \cup T$. Let $S_{m}$ denote the set of successors of $m$. Then

$$
p_{k}\left(S \cup\{m\}-S_{m}, n\right) \leqq 1+p_{k}(S \cup T, n) .
$$

None of the proofs involves any new ideas and we therefore omit them. Theorem 2 now follows by exactly the same argument that led to Theorem 1.

University of California, Los Angeles 